\title{
Precision Medicine and Precision Nursing: The Era of Biomarkers and Precision Health
}

This article was published in the following Dove Press journal:

International Journal of General Medicine

\author{
Nicola lelapi ${ }^{1,2, *}$ \\ Michele Andreucci ${ }^{3, *}$ \\ Noemi Licastro ${ }^{1,4}$ \\ Teresa Faga ${ }^{3}$ \\ Raffaele Grande ${ }^{5}$ \\ Gianluca Buffone ${ }^{6}$ \\ Sabrina Mellace ${ }^{7}$ \\ Paolo Sapienza $\mathbb{1 D}^{5}$ \\ Raffaele Serra (D) ${ }^{1,4}$
}

'Interuniversity Center of Phlebolymphology (CIFL), International Research and Educational Program in Clinical and Experimental Biotechnology, Department of Surgical and Medical Sciences, Magna Graecia University of Catanzaro, Catanzaro, Italy; ${ }^{2}$ Department of Public Health and Infectious Disease, "Sapienza" University of Rome, Rome, Italy; ${ }^{3}$ Department of Health Sciences, Magna Graecia University of Catanzaro, Catanzaro, Italy; ${ }^{4}$ Department of Medical and Surgical Sciences, Magna Graecia University of Catanzaro, Catanzaro, Italy; ${ }^{5}$ Department of Surgery "P. Valdoni", "Sapienza" University of Rome, Rome, Italy; ${ }^{6}$ Department of Vascular Surgery, Health Agency of Trento, Trento, Italy; ${ }^{7}$ Department of Patient's Service, Civic Health Agency of Trento, Trento, Italy

*These authors contributed equally to this work

\begin{abstract}
Precision health, by means of the support of precision medicine and precision nursing, is able to support clinical decision making in order to tailor optimal health-care decisions, around the individual characteristics of patients. The operational arm of precision health is represented by the use of biomarkers that can give useful information about disease susceptibility, exposure, evolution and response to treatment. Omics, imaging and clinical biomarkers are actually studied for their ability to positively impact health-care management. In this article, we try to address the role of biomarkers in the context of modern medicine and nursing with the view of improving patients care.
\end{abstract}

Keywords: precision medicine, precision nursing, biomarkers, precision health, evidence based medicine, evidence based nursing

\section{Introduction}

The term precision medicine (PM) refers to the tailoring of medical management to the individual traits of every single patient, in order to lead health-care management toward the most effective diagnostic, and treatment options for patients, and therefore, improving care quality and disease management. ${ }^{1}$ Nursing clinical practice models are also evolving through individual-centered clinical decision making, and in order to reflect this new era, they are encountering the methodology of precision nursing $(\mathrm{PN})$ that is able to target patients' needs-based nursing care. ${ }^{2} \mathrm{PM}$ and PN are deeply related in the context of precision health $(\mathrm{PH})$, which considers several biological markers, or biomarkers, such as "omics", with other factors such as lifestyle, social characteristics, economic issues, cultural traits, and environmental factors, in order to have information on disease susceptibility, exposure, trait, evolution, and response to treatment. ${ }^{3,4}$ In this context, biomarkers are the operational arm of $\mathrm{PH}$, and the most updated information of these factors will be discussed in the present review.

\section{Search Strategy}

Eligible studies, in order to be selected for our review, were searched using the Cochrane Central Register of Controlled Trials, Clinical Trials.gov, MEDLINE, Embase, and the Cumulative Index to Nursing and Allied Health Literature (CINAHL) Plus. We used a combination of keywords to identify relevant studies. The MEDLINE search strategy below was also adapted as appropriate for other databases: (Precision Medicine) OR (Precision Nursing) OR (Precision Health) OR (Biomarkers) OR (Omics). The last search was run on September 30, 2020. In
Correspondence: Raffaele Serra (CIFL), International Research and Educational Program in Clinical and Experimental Biotechnology, Department of Surgical and Medical Sciences, Magna Graecia University of Catanzaro, Viale Europa,

Catanzaro 88100 , Italy

Tel +39096I3647380

Email rserra@unicz.it
International Journal of General Medicine 2020:|3 |705-|7|I 
addition, in order to search databases, we examined trials registries and we checked reference lists.

\section{Results}

The main findings of the review search are reported below and deal with omics, imaging biomarkers, clinical biomarkers, precision medicine, precision nursing, and examples of clinical application of biomarkers.

\section{Omics}

The term omics refers to several disciplines in biomedical sciences that ends with -omics, such as genomics, transcriptomics, proteomics, and metabolomics. The object of the study of these disciplines ends with -ome, such as genome, transcriptome, proteome and metabolome. Genomics focuses on the composition, function, modification, mapping, and editing of genomes. Transcriptomics studies all the RNA molecules within a cell, otherwise known as the transcriptome. Proteomics explores all the proteins (proteome) in a cell, tissue or organism, and metabolomics is a field of research that focuses on the chemical processes undergone by metabolites that are the end product of cellular processes (metabolome). ${ }^{5}$

In the context of genomics, clinicians may explore patients' genetic information through several tests that have two main elements: a technical element that focuses on identifying which variants the patient shows; and an interpretive element in which the importance of identified variants are evaluated from a clinical point of view in order to manage diseases. ${ }^{6,7}$

Clinical issues that cannot be assessed at the genomics analysis can be evaluated in the transcriptomics analysis. In this way, if these alterations have pathogenetic importance, they could be identified more precisely in order to reflect appropriate treatments. In particular, the transcriptomics supports and complements the genomics evaluation, thus implementing genomics and proteomics in the precision health setting. Moreover, transcriptome analysis, will consider several strategies of analysis, depending on whether the evaluation refers to a single gene (or a small group of genes) or all transcripts of a cell, tissue or organism. ${ }^{8}$

The proteome is quite variable, and differs between cells in a tissue or organism, and changes also during time in response to several endogenous or exogenous factors or stimuli. Proteomics uses not only mass spectrometry, but also other highly sensitive methodologies, such as biosensors, microarrays, nuclear magnetic resonance,
$\mathrm{X}$-ray crystallography, as well as complex bioinformatic analysis. ${ }^{9}$

The aim of metabolomics is to provide detailed characterization of metabolic phenotypes in order to improve the effect of precision medicine at multiple levels, such as the characterization of metabolic alterations that refer to a specific disease, the discovery of new therapeutics target, and the possibility either to diagnose disease or monitor activity of treatments. Moreover, unlike genomics and proteomics analysis, metabolomics presents a significant level of complexity, because it aims to analyze molecules that have highly heterogeneous physical properties and also with diversity of technologies used. ${ }^{10}$

\section{Imaging Biomarkers}

The important development in digital imaging, that nowadays is able to produce high-quality and signal-controlled medical images, generated a broad range of valuable information related to the radiology area. Recently, the impressive advances in the field of medical imaging technologies have made it possible to obtain anatomic, functional, metabolic, elements from images acquired in daily routine clinical practice. Moreover, the important development of computing knowledge allows effective signal modeling and dedicated data processing, that generate a large amount of qualitative and quantitative information that could be finalized in the context of precision health. Modern imaging biomarkers are thus able to be integrated into clinical practice and are characterized by high reliability, technical reproducibility, high precision, and adequate appropriateness. ${ }^{11,12}$ Imaging biomarkers, derived from radiological technologies, such as computerized tomography (CT), electrocardiography (ECG), magnetic resonance (MR), perfusion computerized tomography (pCT), positron emission tomography (PET), ultrasound (US) and many others, are able to inform clinicians on disease detection, characterization and treatment response and quantitative analysis may have good potential to provide physicians with effective medical decision making support, during the management of patients. ${ }^{13}$

\section{Clinical Biomarkers}

Clinical biomarkers refer to a wide subcategory of specific medical signs that represent objective indications of patient's clinical state which can be measured and or assessed accurately during medical physical examination. ${ }^{14}$ For example, a pathognomonic sign is a key clinical element, whose presence indicates the existence in a patient of specific disease 
without any doubt (high specificity), even if the absence of the aforementioned sign cannot definitely exclude the possibility of the disease (low sensibility), and this is a clear limitation of the sign; however, as the presence of a pathognomonic sign is able confirm the disease with certainty, for this reason, a pathognomonic sign serves as a clinical biomarker. ${ }^{15}$ In certain conditions, even the presence of a disease may serve as a clinical biomarker for predicting a more severe clinical course of a concomitant disease. For example, it is known that the presence of cardiovascular disease in COVID-19 patients may influence negatively the clinical outcomes of these patients with poorer prognosis. ${ }^{16}$

\section{Precision Medicine}

Precision medicine describes a standard for health-care delivery strictly related to data, analytics, and information. Precision medicine, and all the advanced technologies that support it, focuses on patient's clinical needs and characteristics, digital health, omics, and other biomedical technologies, data sharing and data science to be clinically effective and successful. A precision medicine environment ideally links patients, providers, clinical laboratories, researchers, and physicians. ${ }^{1}$ The development and use of precision medicine have impacted many diseases and specifically pinpointed several treatments, supporting also technological developments, improving global human health and reducing health care costs. ${ }^{17}$

\section{Precision Nursing}

Nurses have a pivotal role in health care, promoting, protecting, and optimizing health through intimately interconnected and multidisciplinary care of individuals and their families. Integrating biomarkers into nursing research and practice has caused an important advance in nursing science across health-care sciences and caregiving research. Nurses can now use biomarkers to objectively identify health risks and identify more precisely the various mechanisms responsible of adverse clinical conditions, and, in this way, modern nurses may effectively develop, target, and finalize personalized interventions to improve the management of patients. In particular, nursing roles are pivotal in supporting precision health, including precision delivery of medications according to modern concepts of pharmacogenetics. In this way, nurses may involve patients and families in health education, also on the meaning and the utility of omics tests, thus supporting adequate care delivery, and collecting also critical feedback and insight on novel technological applications used during clinical and caring pathways. ${ }^{3,18,19}$

\section{Examples of Clinical Application of Biomarkers}

According to what we wrote in the previous paragraphs, it is not surprising that over 900,000 citations are associated with a MEDLINE search on biomarkers. Cardiovascular disease, cancer and, more in general, chronic diseases, that are also linked to human aging, have been considered optimal fields to study the role of several biomarkers. ${ }^{20}$

Considering the aging process, we can follow consecutive clinical manifestations related to the same pathological process. For example, the studies by Serra et al showed how varicocele in a patient can predict the onset of inguinal hernia and chronic venous disease (CVD) in a more advanced age relating also this progression with extracellular matrix (ECM) alterations due to abnormal matrix metalloproteinase (MMP) activity that can even be measured in the blood as well as pathological tissues. $^{21,22}$ MMP family and other metalloproteinase families, such as a disintegrin and metalloproteinase (ADAM), a disintegrin and metalloproteinase with thrombospondin motifs (ADAMTS) regulating ECM composition, homeostasis, and functions, play major roles in cardiovascular disease onset and progression and some members of these families can be effectively measured in human blood and tissues samples in order to give clinical information in several conditions. Moreover, tissue inhibitors of metalloproteinases (TIMPs) that function by forming a one-to-one complex with target metalloproteinases, are able to irreversibly inactivate them by binding to their catalytic site, and when TIMPs are dysregulated, metalloproteinases may be hyperactivated determining EMC alterations. Even TIMPs activity may be measured in human blood and tissues. ${ }^{23-34}$

Sometimes, a biomarker, such as hyperhomocysteinemia, classically related to acute conditions like venous thromboembolism, in certain circumstances may even predict the onset of chronic conditions, such as chronic venous leg ulcers. ${ }^{35}$

In contrast, in acute clinical settings as those of intensive care units, some biomarkers, such as hypoalbuminemia detected at patient admission, may even predict the onset of pressure ulcers that can complicate a patient's hospital stay. ${ }^{36,37}$ 
The detection of particular forms of biofilm in endovenous devices, such as central venous catheters, may serve as biomarkers predicting particular complications for the implantation and for the functioning of these devices. ${ }^{38}$

Chandler et al, in a prospective study conducted on middle-aged and older women, showed that some lipid biomarkers, such as circulating apo A-I and HDL, were inversely associated with risk of total cancer and lung cancer, whereas others lipid biomarkers, such as triglycerides and apo B-100, were positively associated, and HDL was inversely associated, with the risk of colorectal cancer, suggesting a probable effect of lipid metabolism in the onset of cancer. ${ }^{39}$

MMPs are upregulated at all stages of cancer, including the stage of metastasis, and MMP expression significantly increases at advanced cancer stages. Therefore, several strategies for MMP specific targeting, such as ECM degradation, MMP cleavage, MMP binding, and MMP-induced environmental changes, are currently fields of research activities. $^{40}$ Moreover, MMP-9 expression seems to be related with metastasizing process, survival and recurrence rates, in patients with breast cancer. ${ }^{41}$

Mehri showed that blood gases, maximum consumed oxygen volume $\left(\mathrm{VO}_{2} \mathrm{max}\right)$, cytokines and oxidative stress evaluation may be very useful in nursing diagnosis in clinical practice. ${ }^{42}$

In the nursing area an accurate family health history must be collected by nurses, as this represent a key nursing action that can help identify the need for omics testing for various risk factor and disease conditions. ${ }^{3}$ For example, in oncology setting in the area of hereditary breast and ovarian cancer family history collected by nurses may serve to identify women with an increased risk for harmful genetic mutations. Women with positive screening results should receive genetic counseling and, if appropriate, relative genetic testing. In this context, in the US, the American Nurses Credentialing Center (ANCC) certification in Advanced Genetics Nursing is a new mechanism for documenting genomics competencies that are pivotal for preparing nurses to adequately use these clinical knowledges in health-care settings, in order to contribute to better health care for patients and their families.

\section{Discussion}

The main aim of $\mathrm{PH}$ is to plan disease prevention and health-care interventions considering patient's variability, lifestyle, and omics profile. ${ }^{10,43}$

On the one hand, biomarkers used in $\mathrm{PH}$ may be disease-related to determine the risk of disease, to diagnose a disease, to monitor disease progression and to monitor complication. On the other hand, biomarkers may be intervention-related and may serve as predictors of treatment outcomes, such as those of surgical procedures and they may serve also for drug modulation, predicting and also monitoring drug toxicity. ${ }^{12}$

It is important that physicians and nurses particularly focus on advances in PM and PN in health care. In fact, the impact of biomarkers information and related advanced technology can potentially improve health care outcomes from the point of view of quality, and safety, and can also result in cost savings. Moreover, these goals depend on the optimization of technological resources that support also clinical data storage technology, including the electronic health records of health-care system. In fact, individual omics profile variations may inform the risk of a disease, in all ages and clinical context (including in the prenatal, newborn, infancy, adolescence, and adult ages). Moreover, omics assessment can also be used as a screening evaluation in order to better identify health conditions, improve medication selection, including therapies that may be designed specifically to impact the specific omics profile underlying a disease, and thus tailoring and globally optimizing the management of symptoms. ${ }^{7,44}$

Modern nursing benefit from the inclusion of biomarkers into care pathways, helping nurses to more efficiently administer tailored therapies and globally care for patients, especially those with frailty issues. ${ }^{19,45}$

Nowadays the profession of nursing is a great part, and also an important driving force of health care, and nurse researchers realize that $\mathrm{PH}$ is a priority for advancing nursing science as $\mathrm{PH}$ studies individual variability in personal and environmental patient's own characteristics. In this context, lifestyle, existing comorbidities, cognitive and emotional factors, and frailty issues should be related to omics and other biomarkers. Successful utilization of $\mathrm{PH}$ requires interprofessional collaborations with a range of multidisciplinary elements and in this scenario, PN plays an essential role. Currently, nurses are appropriately prepared and qualified to conduct clinical and translational scientific studies, such as recruiting patients and monitoring health factors and symptoms in interventional settings, thus serving as strong advocates for patients, and their families. While the advent of PH and PN are an effective opportunity for nurses to advance nursing practice and nursing research through important and exclusive challenges in health-care interventions and health innovations, they also demand in the near future efforts in supporting 


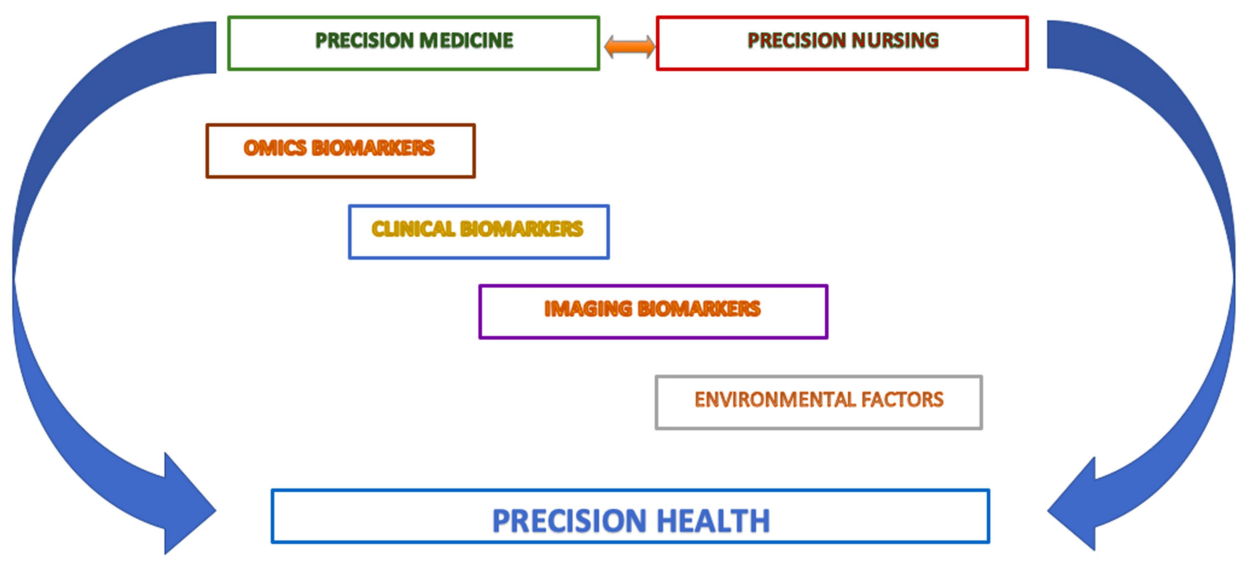

Figure I Elements of precision health.

more specific and dedicated educational pathways, specialized training, and updated knowledge of novel biomarkers in order to incorporate them effectively into daily nursing practice. Precision nursing actually needs longitudinal cohort studies in order to identify which interventions would promote the best health outcomes given patients' particular omics features, together with lifestyle and environmental characteristics. Moreover, solid patient outcome measurement systems need to be investigated and validated in order to evaluate effective PN efficacy at several health care levels (health-care provider, clinic, hospital, home care). ${ }^{3,19,46}$ In this view, PM and PN follow the aims of $\mathrm{PH}$ that is a modern way of clinical thinking and caring patients, that is able to link the patient's characteristics with the omics profile, and with the environmental profile (such as lifestyle, socioeconomic issues, etc) in order to improve strategies for disease prevention and treatment in a new context of holistic care (Figure 1). ${ }^{47}$

\section{Conclusion}

Governments and National Health Systems have a central role to play in $\mathrm{PH}$, and they have the task to turn the actual knowledge on biomarkers into evidence-based reality, supporting health systems and subsequent environmental changes. In fact, the implementation of $\mathrm{PH}$ will be used for more effective decision making in medicine and public health to improve quality of life. ${ }^{48}$

In conclusion, precision medicine and precision nursing, in the context of $\mathrm{PH}$ and among the current options to manage health, are the most innovative and effective tools that can be used to promote health, well-being, and to deliver optimal health care.

\section{Funding}

This research received no specific grant from any funding agency in the public, commercial or not-for-profit sectors.

\section{Disclosure}

The authors report no conflicts of interest in this work.

\section{References}

1. Ginsburg GS, Phillips KA. Precision medicine: from science to value. Health Aff (Millwood). 2018;37(5):694-701. doi:10.1377/ hlthaff.2017.1624

2. Yuan C. Precision nursing: new era of cancer care. Cancer Nurs. 2015;38(5):333-334. doi:10.1097/NCC.0000000000000290

3. Fu MR, Kurnat-Thoma E, Starkweather A, et al. Precision health: a nursing perspective. Int J Nurs Sci. 2019;7(1):5-12.

4. Serra R, Ielapi N, Barbetta A, Andreucci M, de Franciscis S. Novel biomarkers for cardiovascular risk. Biomark Med. 2018;12 (9):1015-1024. doi: $10.2217 / \mathrm{bmm}-2018-0056$

5. Vailati-Riboni M, Palombo V, Loor JJ. What are omics sciences? In: Ametaj B, editor. Periparturient Diseases of Dairy Cows. Cham: Springer; 2017. doi:10.1007/978-3-319-43033-1_1.

6. Aronson SJ, Rehm HL. Building the foundation for genomics in precision medicine. Nature. 2015;526(7573):336-342. doi:10.1038/ nature 15816

7. McCormick KA, Calzone KA. The impact of genomics on health outcomes, quality, and safety. Nurs Manage. 2016;47(4):23-26. doi:10.1097/01.NUMA.0000481844.50047.ee

8. Khodadadian A, Darzi S, Haghi-Daredeh S, et al. Genomics and transcriptomics: the powerful technologies in precision medicine. Int J Gen Med. 2020;13:627-640. doi:10.2147/IJGM.S249970

9. Nice EC. The status of proteomics as we enter the 2020s: towards personalised/precision medicine. Anal Biochem. 2020;2020:113840. doi:10.1016/j.ab.2020.113840

10. Clish CB. Metabolomics: an emerging but powerful tool for precision medicine. Cold Spring Harb Mol Case Stud. 2015;1(1):a000588. doi:10.1101/mcs.a000588

11. Martí-Bonmatí L. Introduction to the stepwise development of imaging biomarkers. In: Martí-Bonmatí L, Alberich-Bayarri A, editors. Imaging Biomarkers. Cham: Springer; 2017. doi:10.1007/978-3-31943504-6_2. 
12. Herold CJ, Lewin JS, Wibmer AG, et al. Imaging in the age of precision medicine: summary of the proceedings of the 10th biannual symposium of the international society for strategic studies in radiology. Radiology. 2016;279(1):226-238. doi:10.1148/ radiol.2015150709

13. deSouza NM, Achten E, Alberich-Bayarri A, et al. Validated imaging biomarkers as decision-making tools in clinical trials and routine practice: current status and recommendations from the EIBALL* subcommittee of the European society of radiology (ESR). Insights Imaging. 2019;10(1):87. doi:10.1186/s13244-019-0764-0

14. Strimbu K, Tavel JA. What are biomarkers? Curr Opin HIV AIDS. 2010;5(6):463-466. doi:10.1097/COH.0b013e32833ed177

15. Janeway EG. Limitations of pathognomonic signs and symptoms. JAMA. 1884;III(5):116-120. doi:10.1001/jama.1884.02390540004001a

16. Ielapi N, Licastro N, Provenzano M, Andreucci M, Franciscis S, Serra R. Cardiovascular disease as a biomarker for an increased risk of COVID-19 infection and related poor prognosis. Biomark Med. 2020;14(9):713-716. doi:10.2217/bmm-2020-0201

17. Slikker W Jr. Biomarkers and their impact on precision medicine. Exp Biol Med (Maywood). 2018;243(3):211-212. doi:10.1177/ 1535370217733426

18. Corwin EJ, Ferranti EP. Integration of biomarkers to advance precision nursing interventions for family research across the life span. Nurs Outlook. 2016;64(4):292-298. doi:10.1016/j.outlook.2016.04.007

19. Richmond ES, Dunn D. Biomarkers: an overview for oncology nurses. Semin Oncol Nurs. 2012;28(2):87-92. doi:10.1016/j. soncn.2012.03.002

20. Rivero-Segura NA, Bello-Chavolla OY, Barrera-Vázquez OS, Gutierrez-Robledo LM, Gómez-Verjan JC. Promising biomarkers of human aging: in search of a multi-omics panel to understand the aging process from a multidimensional perspective. Ageing Res Rev. 2020;2020:101164. doi:10.1016/j.arr.2020.101164

21. Serra R, Buffone G, Costanzo G, et al. Varicocele in younger as risk factor for inguinal hernia and for chronic venous disease in older: preliminary results of a prospective cohort study. Ann Vasc Surg. 2013;27(3):329-331. doi:10.1016/j.avsg.2012.03.016

22. Serra R, Buffone G, Costanzo G, et al. Altered metalloproteinase- 9 expression as least common denominator between varicocele, inguinal hernia, and chronic venous disorders. Ann Vasc Surg. 2014;28 (3):705-709. doi:10.1016/j.avsg.2013.07.026

23. Metzinger L, de Franciscis S, Serra R. The management of cardiovascular risk through epigenetic biomarkers. Biomed Res Int. 2017;2017:9158572. doi:10.1155/2017/9158572

24. de Franciscis S, Metzinger L, Serra R. Roles and clinical applications of biomarkers in cardiovascular disease. Biomed Res Int. 2016;2016:8982796. doi:10.1155/2016/8982796

25. de Franciscis S, Metzinger L, Serra R. The discovery of novel genomic, transcriptomic, and proteomic biomarkers in cardiovascular and peripheral vascular disease: the state of the art. Biomed Res Int. 2016;2016:7829174. doi:10.1155/2016/7829174

26. de Franciscis S, Gallelli L, Amato B, et al. Plasma MMP and TIMP evaluation in patients with deep venous thrombosis: could they have a predictive role in the development of post-thrombotic syndrome? Int Wound J. 2016;13(6):1237-1245. doi:10.1111/iwj.12489

27. de Franciscis S, De Caridi G, Massara M, et al. Biomarkers in post-reperfusion syndrome after acute lower limb ischaemia. Int Wound J. 2016;13(5):854-859. doi:10.1111/iwj.12392

28. Serra R, Grande R, Montemurro R, et al. The role of matrix metalloproteinases and neutrophil gelatinase-associated lipocalin in central and peripheral arterial aneurysms. Surgery. 2015;157(1):155-162. doi:10.1016/j.surg.2014.06.008

29. Serra R, Gallelli L, Butrico L, et al. From varices to venous ulceration: the story of chronic venous disease described by metalloproteinases. Int Wound J. 2017;14(1):233-240. doi:10.1111/ iwj. 12594
30. Provenzano M, Andreucci M, Garofalo C, et al. The association of matrix metalloproteinases with chronic kidney disease and peripheral vascular disease: a light at the end of the tunnel? Biomolecules. 2020;10(1):154. doi:10.3390/biom10010154

31. Busceti MT, Grande R, Amato B, et al. Pulmonary embolism, metalloproteinases and neutrophil gelatinase associated lipocalin. Acta Phlebol. 2013;14(3):115-121.

32. Serra R, Gallelli L, Grande R, et al. Hemorrhoids and matrix metalloproteinases: a multicenter study on the predictive role of biomarkers. Surgery. 2016;159(2):487-494.

33. Serra R, Ielapi N, Barbetta A, et al. Biomarkers for precision medicine in phlebology and wound care: a systematic review. Acta Phlebol. 2017;18(2):52-56.

34. Serra R, Barbetta A, Ielapi N, De Francisci S, Gasbarro V. Current knowledge on venous and lymphatic ulcers. A systematic review on evidence-based medicine. Acta Phlebol. 2017;18(2):47-51.

35. de Franciscis S, De Sarro G, Longo P, et al. Hyperhomocysteinaemia and chronic venous ulcers. Int Wound J. 2015;12(1):22-26. doi:10.1111/iwj.12042

36. Serra R, Caroleo S, Buffone G, et al. Low serum albumin level as an independent risk factor for the onset of pressure ulcers in intensive care unit patients. Int Wound J. 2014;11(5):550-553. doi:10.1111/ iwj. 12004

37. Serra R, Grande R, Buffone G, et al. Albumin administration prevents the onset of pressure ulcers in intensive care unit patients. Int Wound J. 2015;12(4):432-435. doi:10.1111/iwj.12131

38. Ielapi N, Nicoletti E, Lorè C, et al. The role of biofilm in central venous catheter related bloodstream infections: evidence-based nursing and review of the literature. Rev Recent Clin Trials. 2020;15 (1):22-27. doi:10.2174/1574887114666191018144739

39. Chandler PD, Song Y, Lin J, et al. Lipid biomarkers and long-term risk of cancer in the women's health study. Am J Clin Nutr. 2016;103 (6):1397-1407. doi:10.3945/ajcn.115.124321

40. Isaacson KJ, Martin Jensen M, Subrahmanyam NB, Ghandehari H. Matrix-metalloproteinases as targets for controlled delivery in cancer: an analysis of upregulation and expression. $J$ Control Release. 2017;259:62-75. doi:10.1016/j.jconrel.2017.01.034

41. Yousef EM, Tahir MR, St-Pierre Y, Gaboury LA. MMP-9 expression varies according to molecular subtypes of breast cancer. $B M C$ Cancer. 2014;14(1):609. doi:10.1186/1471-2407-14-609

42. Mehri SN. Biomarkers-based research in nursing. Iran J Crit Care Nurs. 2009;2(3):121-125.

43. Serra R, Ssempijja L, Provenzano M, Andreucci M. Genetic biomarkers in chronic venous disease. Biomark Med. 2020;14(2):75-80. doi:10.2217/bmm-2019-0408

44. Kellogg RA, Dunn J, Snyder MP. Personal omics for precision health. Circ Res. 2018;122(9):1169-1171. doi:10.1161/ CIRCRESAHA.117.310909

45. Ielapi N, Licastro N, Catana M, Bracale UM, Serra R. Vascular nursing and vascular surgery. Ann Vasc Surg. 2020;68:522-526. doi:10.1016/j.avsg.2020.05.038

46. Hacker ED, McCarthy AM, DeVon H. Precision health: emerging science for nursing research. Nurs Outlook. 2019;67(4):287-289. doi:10.1016/j.outlook.2019.06.008

47. Dickson C, Hyppönen E. Precision health: a primer for physiotherapists. Physiotherapy. 2020;107:66-70. doi:10.1016/j. physio.2019.08.004

48. Whitsel LP, Wilbanks J, Huffman MD, Hall JL. The role of government in precision medicine, precision public health and the intersection with healthy living. Prog Cardiovasc Dis. 2019;62(1):50-54. doi:10.1016/j.pcad.2018.12.002 


\section{Publish your work in this journal}

The International Journal of General Medicine is an international, peer-reviewed open-access journal that focuses on general and internal medicine, pathogenesis, epidemiology, diagnosis, monitoring and treatment protocols. The journal is characterized by the rapid reporting of reviews, original research and clinical studies across all disease areas. The manuscript management system is completely online and includes a very quick and fair peer-review system, which is all easy to use. Visit http://www.dovepress.com/ testimonials.php to read real quotes from published authors.

Submit your manuscript here: https://www.dovepress.com/international-journal-of-general-medicine-journal 